\title{
Association between Diabetes Mellitus and Oral Health Status in Japanese Adults
}

\author{
Masayuki Ueno ${ }^{1 *}$, Susumu Takeuchi ${ }^{1}$, Akiko Oshiro ${ }^{1}$, Kayoko Shinada ${ }^{1}$, Satoko Ohara ${ }^{2}$, Yoko Kawaguchi ${ }^{1}$ \\ ${ }^{1}$ Department of Oral Health Promotion, Graduate School of Medical and Dental Sciences, Tokyo Medical and Dental \\ University, Tokyo, Japan \\ ${ }^{2}$ Department of Comprehensive Oral Health Care, Faculty of Dentistry, Tokyo Medical and Dental University, Tokyo, Japan
}

\begin{abstract}
Aim The objective of this study was to analyze the oral health among Japanese adults, with and without diabetes mellitus.

Methodology The subjects were 518 community residents aged 20 to 91 years in Japan, who participated in the "Akita health and nutrition survey" and the "Akita dental disease survey", conducted in 2006. The surveys comprised a self-administered questionnaire, along with medical and dental examinations.
\end{abstract}

Results Using the community periodontal index (CPI), the mean numbers of sextants presenting codes 0,1 and 2 were significantly lower in diabetics than non-diabetics among the 59 years or younger age group. Although the mean numbers of sextants with codes 0,1 and 2 among the 60 to 69 years age group were lower, and sextants with a code $X$ among the 59 years or younger age group were higher in diabetics than non-diabetics, no statistically significant differences were detected. The tendency of lower mean numbers of natural teeth and functional tooth units in diabetics than non-diabetics was observed, however no differences were statistically significant.

Conclusion The outcome of periodontal disease seemed to be influenced by the diabetic state to some degree, but a clear association between diabetes and oral health status was not found.

Keywords diabetes mellitus, periodontal disease, community periodontal index, functional tooth units

Received Jan 25, 2010; Revision accepted Feb. 25, 2010

\section{Introduction}

Diabetes mellitus is very prevalent, affecting over 217 million people worldwide, and its prevalence is expected to increase in the future (Nelson, 2008). According to the World Health Organization, at least 366 million people around the world are projected to have diabetes mellitus by the year 2030 (Smyth and Heron, 2006). Periodontal disease, which is one of the most common dental problems and a major cause of tooth loss, is caused by a variety of oral plaque bacteria. In Japan, approximately $70 \%$ of the population aged 15 years or older has some degree of periodontal problems and more than $20 \%$ of the population aged 40 years or older have periodontal pockets
$4 \mathrm{~mm}$ or deeper (Statistical analysis committee on the survey of dental diseases, 2005).

Studies establishing the relationship among diabetes mellitus, periodontal health and subsequent tooth loss have been broadly reported (Campus et al., 2005; Khader et al., 2006; Furukawa et al., 2007; Taylor and Borgnakke, 2008). There is ample evidence of the biological and epidemiological links between periodontal disease and diabetes, especially, type 2 diabetes mellitus.

The postulated mechanism for diabetic effects on periodontal disease is that diabetes-enhanced inflammation and apoptosis specifically affect periodontal tissues (Graves et al., 2007). Moreover, the increased severity of periodontal disease in diabetes mellitus may reflect an alteration in the 
pathogenic potential of bacteria, enhancing a breakdown of periodontal tissues (Southerland et al., 2006; Nishimura et al., 2007; Ebersole et al., 2008), resulting in more frequent and severe periodontal-tissue destruction.

Periodontal diseases also affect the metabolic state in diabetes mellitus. Chronic gram-negative anaerobic bacteria in periodontal infections possibly influence poor glycemic control and increase the risk of diabetes complications (Genco et al., 2005; Mealey and Ocampo, 2007; Taylor et al., 2008).

In epidemiological studies, periodontal impairment is significantly more frequent and severe in subjects with type 2 diabetes mellitus (Tsai et al., 2002; Lalla et al., 2004; Gursoy et al., 2008). The National Health and Nutrition Examination Survey III found that individuals with poorly-controlled type 2 diabetes mellitus had a significantly higher prevalence of severe periodontitis than those without diabetes after controlling for age, education, smoking, and amounts of calculus (Tsai et al., 2002). Furthermore, diabetic patients may be more likely to lose teeth than non-diabetic patients (Campus et al., 2005; Furukawa et al., 2007).

Several reports detected, however, no significant differences in levels of periodontal disease between subjects with and without diabetes mellitus (ArrietaBlanco et al., 2003; Ogunbodede et al., 2005). Therefore, interpretation of research findings should be made with caution because of differences in the types and numbers of subjects, research design, and definition of diabetes mellitus, periodontal parameters and other clinical conditions.

Many reports have investigated the relationship between diabetes mellitus and periodontal disease, but none have examined the possible influence of diabetes mellitus on the loss of posterior teeth, often evaluated with indexes like Functional Tooth Units (FTUs) (Hatch et al., 2001; Ueno et al., 2008; Ueno et al., 2010).

Our hypothesis is that diabetic subjects will have fewer natural teeth due to progressive periodontal disease, and consequently have fewer occluding posterior tooth pairs compared to non-diabetics. The objective of this cross-sectional study was to analyze the oral health status (i.e., level of periodontal disease, number of natural teeth and FTUs) among Japanese residents with and without diabetes mellitus using survey data collected by the health and welfare section of the Akita prefectural government.

\section{Materials and Methods}

\section{Subjects}

The subjects were adult residents of Akita prefecture, Japan, selected using a multi-level, stratified sampling method based on the national census. The numbers of subjects who participated in the "Akita health and nutrition survey", the "Akita dental disease survey" and both surveys were 1696,936 and 589, respectively. The final sample used for the analysis consisted of 518 subjects (201 men and 317 women) aged 20 to 91 years drawn from those who had completed both surveys, after excluding those with missing data. The subjects all underwent a self-administered questionnaire, a medical and dental examination between October and December of 2006.

\section{Questionnaire}

The self-administered questionnaire included demographic (i.e., age and gender) and smoking status information (i.e., non-smoker, past-smoker and current-smoker).

\section{Medical examination}

Subjects undertook a routine medical examination, including a blood chemical analysis for hemoglobin A1c (HbA1c) level. The following criteria of the Japanese Ministry of Health, Labour and Welfare were used to diagnose diabetes: 1) A HbAlc $\geqslant 6.1,2$ ) use of insulin, or 3) use of hypoglycemic medicines.

\section{Dental examination}

Clinical examinations were conducted, which included the assessment of community periodontal index (CPI) (Namariyama, 2008), number of teeth (third molars were excluded from the analysis), number of FTUs, and the oral hygiene condition of the subjects. All dental examinations were performed by dentists from the local dental association, using 
a dental mirror and a CPI probe. Standardized clinical criteria were described in detail in a handbook distributed to all participating dentists. The CPI index was used for the periodental assessment, in which the dentition was divided into sextants. The highest CPI code was recorded in each segment (code 0: no signs of periodontal disease, code 1: gingival bleeding after gentle probing, code 2: supragingival or subgingival calculus, code $3: 4$ to $5 \mathrm{~mm}$ deep pathologic pockets, code 4: $6 \mathrm{~mm}$ or deeper pathologic pockets and code $X$ : missing index teeth). Periodontal status was divided into three categories according to the CPI code: 0,1 and 2, 3 and 4, and $X$.

The FTUs were defined as pairs of occluding posterior natural teeth (i.e., sound, restored and $\mathrm{D}_{1}-\mathrm{D}_{4}$ scale carious teeth) and artificial teeth on implant-supported, fixed (bridge pontics) or removable prostheses (Ogunbodede et al., 2005). $\mathrm{D}_{4}$ scale carious teeth with extensive coronal destruction and missing teeth were regarded as non-functional. Two occluding premolars were defined as one FTU, and two occluding molars were defined as two FTUs. Therefore, a person with a complete dentition had 12 FTUs (third molars being excluded).

The oral hygiene condition was visually evaluated by examining all teeth present without using disclosing solution as: 1) good, plaque covering less than one-third of tooth surfaces; 2) fair, plaque covering more than one-third but less than two-thirds of tooth surfaces; and, 3) poor, plaque covering more than two-thirds of tooth surfaces. The worst score was recorded as representative for that subject.

\section{Statistical analysis}

The prevalence of diabetes mellitus was calculated for three age groups: 59 years or younger, 60 to 69 years, and 70 years or older. Subsequent analyses were conducted over these three age groups. The number of sextants by the three CPI code categories was compared between diabetic and nondiabetic subjects, using a Mann-Whitney's U test. The number of natural teeth and FTUs were positively skewed because of a high proportion of zero values. Testing with the Shapiro-Wilk W found that neither the number of natural teeth nor
FTUs were normally distributed. Therefore, we used a generalized linear model (GLM) of the negative binominal distribution with a logit builtin link function (Lewsey and Thomson, 2004; Javali and Parameshwar 2008). For the GLM, the numbers of natural teeth and FTUs were treated as response variables and the diabetic status as an explanatory variable. To control for confounding factors, three dichotomous dummy variables were created for covariates: sex (male, female), oral hygiene condition (poor, fair or good) and smoking status (non-smokers, past-smokers or current-smokers). The SPSS statistical package, version 17.0J (SPSS Japan Inc., Tokyo, Japan) was used for all the statistical analyses.

\section{Ethics}

This study protocol was approved by the Tokyo Medical and Dental University Ethical Committee (No. 361).

\section{Results}

\section{Characteristics of subjects by age group}

As shown in Table 1, characteristics of subjects in each age group are similar. The proportion of female was higher than that of male. Most subjects were non-smokers. Mean $\mathrm{HbA1c}$ ranged from 5.18 to 5.56. The greatest proportion of subjects was that with fair oral hygiene conditions.

\section{Prevalence of diabetes mellitus}

A total of 48 people $(9.3 \%)$ were diagnosed as having type 2 diabetes mellitus according to the medical examination and previously mentioned criteria. The proportion of subjects with diabetes mellitus increased with age group: $4.0 \%$ in the 59 years or younger age group, $13.3 \%$ in the 60 to 69 years age group, and $14.9 \%$ in the 70 years or older age group. The prevalence in the 59 years or younger age group was significantly lower than that in the 60 to 69 years age group $(P<0.01)$ and 70 years or older age group $(P<0.001)$ (Table 2$)$. The prevalence between males and females did not differ significantly in any age group. 
Table 1 Characteristics of subjects by age group

\begin{tabular}{|c|c|c|c|c|}
\hline \multirow[b]{3}{*}{ Variable } & \multirow[b]{3}{*}{ Category/Range } & \multicolumn{3}{|c|}{ Age group } \\
\hline & & $\leqslant 59$ & $60-69$ & $\geqslant 70$ \\
\hline & & N/Mean (\%/SD) & N/Mean (\%/SD) & N/Mean (\%/SD) \\
\hline \multirow[t]{2}{*}{ Gender } & Male & 89 (35.7) & $58(45.3)$ & $54(38.3)$ \\
\hline & Female & $160(64.3)$ & $70(54.7)$ & $87(61.7)$ \\
\hline \multirow[t]{3}{*}{ Smoking status } & Non-smoker & $169(67.9)$ & $94(73.4)$ & $116(82.3)$ \\
\hline & Past-smoker & $27(10.8)$ & $18(14.1)$ & $9(6.4)$ \\
\hline & Current-smoker & $53(21.3)$ & $16(12.5)$ & $16(11.3)$ \\
\hline $\mathrm{HbA} 1 \mathrm{c}$ & $4.3-10.3$ & $5.18(0.39)$ & $5.56(0.87)$ & $5.50(0.66)$ \\
\hline \multirow[t]{3}{*}{ Oral hygiene condition } & Good & $45(18.1)$ & $16(12.5)$ & $11(7.8)$ \\
\hline & Fair & $140(56.2)$ & $68(53.1)$ & $94(66.7)$ \\
\hline & poor & $64(25.7)$ & $44(34.4)$ & $36(25.5)$ \\
\hline
\end{tabular}

Table 2 Frequency of subjects with diabetes mellitus

\begin{tabular}{cccc}
\hline & Diabetic & Non-diabetic & Total \\
\cline { 2 - 4 } Age group & $N(\%)$ & $N(\%)$ & $N$ \\
\hline$\leqslant 59$ & $10(4.0)$ & $239(96.0)$ & 249 \\
$60-69$ & $17(13.3)$ & $111(86.7)$ & 128 \\
$\geqslant 70$ & $21(14.9)$ & $120(85.1)$ & 141 \\
Total & $48(9.3)$ & $470(90.7)$ & 518 \\
\hline
\end{tabular}

\section{Periodontal condition}

The mean number of sextants with CPI codes of 0,1 and 2 decreased with age group with or without diabetes (Table 3 ). The means were lower in diabetics than non-diabetics in the 59 years or younger age group $(P<0.05)$ and the 60 to 69 years age group $(P=0.09)$. In contrast, the mean number of sextants with CPI codes of 3 and 4 increased with age group, and the means were higher in diabetics than non-diabetics, except for the 70 years or older age group. Similarly, the mean number of sextants with a CPI code of X increased with age group in both diabetics and non-diabetics, and the means were higher in diabetics than non-diabetics, particularly in the 59 years or younger age group and approached statistical significance $(P=0.08)$.

\section{Number of natural teeth}

The mean number of natural teeth (after adjusting for sex, oral hygiene condition and smoking status) decreased with age in both diabetics and non-diabetics (Table 4). The means tended to be lower in diabetics than non-diabetics in every age group. The differences in mean number of natural teeth between diabetics and non-diabetics were 1.44 in the 59 years or younger age group, 0.92 in the 60 to 69 years age group, and 0.88 in the 70 years or older age group, but again no differences were statistically significant.

Table 3 Mean number of sextants by CPI code in subjects by age group

\begin{tabular}{|c|c|c|c|c|c|c|c|c|c|}
\hline \multirow{4}{*}{$\begin{array}{l}\text { CPI } \\
\text { code }\end{array}$} & \multicolumn{9}{|c|}{ Age group } \\
\hline & \multicolumn{3}{|c|}{$\leqslant 59$} & \multicolumn{3}{|c|}{$60-69$} & \multicolumn{3}{|c|}{$\geqslant 70$} \\
\hline & Diabetic & Non-diabetic & & Diabetic & Non-diabetic & & Diabetic & Non-diabetic & \\
\hline & Mean (SE) & Mean (SE) & $P$ & Mean (SE) & Mean (SE) & $P$ & Mean (SE) & Mean (SE) & $P$ \\
\hline $0,1,2$ & $3.00(0.68)$ & $4.43(0.12)$ & $0.03^{*}$ & $1.82(0.49)$ & $2.78(0.21)$ & 0.09 & $1.29(0.31)$ & $1.36(0.17)$ & 0.64 \\
\hline 3,4 & $1.70(0.70)$ & $1.02(0.10)$ & 0.43 & $1.77(0.42)$ & $1.16(0.13)$ & 0.17 & $0.57(0.22)$ & $0.87(0.13)$ & 0.40 \\
\hline$x$ & $1.30(0.54)$ & $0.56(0.07)$ & 0.08 & $2.41(0.56)$ & $2.06(0.20)$ & 0.55 & $4.14(0.41)$ & $3.77(0.21)$ & 0.62 \\
\hline
\end{tabular}

*Statistically significant at $P<0.05$. 
Table 4 Adjusted mean number of natural teeth and FTUs

\begin{tabular}{|c|c|c|c|c|c|c|}
\hline \multirow[b]{3}{*}{ Age group } & \multicolumn{3}{|c|}{ Number of natural teeth } & \multicolumn{3}{|c|}{ Number of FTUs } \\
\hline & Diabetic & Non-diabetic & & Diabetic & Non-diabetic & \\
\hline & Mean (SE) & Mean (SE) & $P$ & Mean (SE) & Mean (SE) & $P$ \\
\hline$\leqslant 59$ & $22.76(7.43)$ & $24.20(1.60)$ & 0.85 & $9.02(3.05)$ & $9.86(0.67)$ & 0.79 \\
\hline $60-69$ & $16.69(4.24)$ & $17.61(1.72)$ & 0.84 & $9.58(2.47)$ & $9.80(0.98)$ & 0.94 \\
\hline$\geqslant 70$ & $9.00(2.10)$ & $9.88(0.95)$ & 0.70 & $9.68(2.24)$ & $10.04(0.96)$ & 0.88 \\
\hline
\end{tabular}

Adjusted for sex, oral hygiene condition and smoking status.

\section{Number of FTUs}

The mean number of FTUs (after adjusting for sex, oral hygiene condition and smoking status) increased slightly with age group in diabetics, but not in non-diabetics. The mean number of FTUs were somewhat lower in diabetics than nondiabetics in all age groups $(0.84$ in the 59 years or younger age group, 0.22 in the 60 to 69 years age group and 0.36 in the 70 years or older age group), but no differences were statistically significant.

\section{Discussion}

\section{Prevalence of diabetes mellitus}

According to the Japanese survey of diabetes mellitus conducted in 2002, approximately 740000 people had diabetes mellitus, and $98 \%$ of these cases were type 2 diabetes mellitus. The number of people with diabetes mellitus increased with age group: $4.3 \%$ in people aged 59 years or younger, $14.4 \%$ in those 60 to 69 years, and $15.7 \%$ in those 70 years or older (Ministry of health, labour and welfare, 2004). These percentages are very similar to those in the current sample; therefore, the subjects in this study are considered to likely represent the general Japanese population regarding diabetes mellitus prevalence.

\section{Diabetes and periodontal disease}

Our results indicate a probable relationship between type 2 diabetes mellitus and periodontal disease, and a tendency for diabetics to have fewer natural teeth and FTUs than non-diabetics. Because the prevalence of periodontal disease increases and the number of natural teeth decreases steadily with age, data were analyzed by age group.

Measurements of pocket depth and bleeding on probing were not performed in this survey. Instead the original dental survey used the CPI method as it was a community-based mass examination. The subjects with CPI codes of 0,1 and 2 were regarded as healthy or with gingivitis, those with CPI codes of 3 and 4 were regarded as having periodontitis, and those with a code of $X$ reflected missing index teeth.

Diabetics tended to have fewer sextants with CPI codes of 0,1 and 2 and more sextants with codes of 3 and 4 or $X$ than non-diabetics. This tendency was more noticeable in the younger age groups. This difference was not obvious in subjects aged 70 years or older. This is partly because more than $60 \%$ of sextants were missing in both diabetics and non-diabetics in this age group. A previous study conducted in Japan, using the community periodontal index of treatment needs (CPITN), showed that no diabetic patients had a CPI code of 0 , and more than $90 \%$ of diabetic patients had CPI codes of 3 and 4 compared to $56 \%$ of non-diabetic subjects (Ohtake et al., 2005). Another study found significantly greater numbers of CPI code 4 in diabetics than in control subjects (Bacic et al., 1988). Hence, the current results support previous conclusions that diabetes mellitus is associated with periodontal disease, and that the outcome of periodontal destruction is significantly more frequent and severe in subjects with diabetes mellitus.

Several studies found, however, no significant association between diabetes mellitus and CPI (Chuang et al., 2005). Chuang et al. reported no significant difference in the CPI index between diabetic and non-diabetic groups. Another study 
found no significant differences in the CPITN between diabetics and controls (Hatch et al., 2001). Differences in types of subjects (i.e., race, gender, and age) and degree of diabetes or other medical conditions might have produced these conflicting results.

\section{Diabetes and numbers of natural teeth and FTUs}

After adjusting for several potentially important risk factors, such as sex, oral hygiene condition and smoking status, the difference in the mean number of natural teeth was approximately one tooth between diabetics and non-diabetics. The difference was not statistically significant and smaller than that reported in a previous study (Campus et al., 2005) in Italy, which found that diabetics had approximately four fewer teeth than non-diabetics. In an earlier study (Leong et al., 2007), diabetic adults had, on average, approximately one more missing tooth than non-diabetics, a number similar to our results. Neither of these previous studies, however, took into account the confounding factors listed above. One study has reported that the number of teeth decreased with increasing HbAlc values (Furukawa et al., 2007), although the mechanism was not known. The HbAlc value of subjects in the former Japanese study by Furukawa et al. was 7.8, similar to the value of 7.0 in the present study. Differences in HbAlc level might influence differences in the number of missing teeth.

The number of occluding posterior tooth pairs (FTUs) are an important index for dietary intake and masticatory performance, which in turn are important factors for activities of daily living and quality of life (Ogunbodede et al., 2005; ArrietaBlanco et al., 2003). A smaller number of FTUs is associated with chewing difficulties, poor dental functional status and negative general health (Shinkai et al., 2001; Kwok et al., 2004). Diabetics tended to have fewer FTUs compared to nondiabetics in this study. Such a tendency was more apparent in the younger age groups. The difference was, on average, approximately one FTU in subjects aged 59 years or younger, corresponding to one pair of occluding premolars. The difference, however, was not statistically significant, and this would be partly because of the lack of power due to the small number of diabetic subjects. In addition, the missing teeth caused by other than periodontal disease could confound the results.

Even though a clear relationship between diabetes mellitus and periodontal disease was not observed, the outcomes of periodontal disease seemed to be influenced by the diabetic state to some degree. Our hypothesis that the deterioration of periodontal health and subsequent tooth loss leads to a decreased number of posterior occluding tooth pairs was not verified in this research. Because the current results were obtained from a cross-sectional study of a small sample of diabetics, it is difficult to detect significant differences and make causal inferences. In any case, the CPI does not really measure periodontal condition, unlike studies based on the pocket depth or gingival bleeding measurement.

A more rigorous and systematic study, using a large population, will be necessary to confirm the current observations. Moreover, prospective or interventional studies are required to clarify the causal association between diabetes mellitus and periodontal disease. Because the subjects in this survey were community residents, not special patients who were often used in other studies, the observed association between diabetes mellitus and periodontal disease in the present study has implications for the prevention of periodontal disease initiation and progression, not only in people with diabetes mellitus, but the general Japanese adult as well.

Dental professionals should more actively utilize medically and dentally related data in order to investigate the relationship between oral and general health. Doing so will allow more efficient examination of medically and dentally related problems and more readily promote interdisciplinary research.

\section{Conclusion}

The outcome of periodontal disease seemed to be influenced by the diabetic state to some degree, but a clear association between diabetes and oral health status was not found in Japanese adults. More rigorous and systematic studies, such as 
prospective or interventional studies, using a large population, will be necessary to confirm the association between diabetes mellitus and periodontal disease.

\section{References}

Arrieta-Blanco J, Bartolomé-Villar B, Jiménez-Martinez E, Saavedra-Vallejo P, Arrieta-Blanco FJ (2003). Dental problems in patients with diabetes mellitus ( II ): gingival index and periodontal disease. Med Oral, 8(4): 233-247.

Bacic M, Plancak D, Granic M (1988). CPITN assessment of periodontal-disease in diabetic-patients. J Periodontol, 59(12): 816-822.

Campus G, Salem A, Uzzau S, Baldoni E, Tonolo G (2005). Diabetes and periodontal disease: a case-control study. J Periodontol, 76(3): 418-425.

Chuang SF, Sung JM, Kuo SC, Huanf JJ, Lee SY (2005). Oral and dental manifestations in diabetic and nondiabetic uremic patients receiving hemodialysis. Oral Surg Oral Med Oral Pathol Oral Radiol Endod, 99(6): 689-695.

Ebersole JL, Holt SC, Hansard R, Novak MJ (2008). Microbiologic and immunologic characteristics of periodontal disease in Hispanic Americans with type 2 diabetes. J Periodontol, 79(4): 637-646.

Furukawa T, Wakai K, Yamanouchi K, Oshida Y, Miyao M, Watanabe T, et al. (2007). Associations of periodontal damage and tooth loss with atherogenic factors among patients with type 2 diabetes mellitus. Intern Med, 46(17): 1359-1364.

Genco RJ, Grossi S, Ho A, Nishimura F, Murayama Y. (2005). A proposed model linking inflammation to obesity, diabetes, and periodontal infections. J Periodontol, 76(11): 2075-2084.

Graves DT, Liu R, Oates TW (2007). Diabetes-enhanced inflammation and apoptosis - impact on periodontal pathosis. Periodontology 2000, 45: 128-137.

Gursoy UK, Marakoglu I, Oztop AY (2008). Relationship between neutrophil functions and severity of periodontitis in obese and/or type 2 diabetic chronic periodontitis patients. Quint Int, 39(6): 485-489.

Hatch J, Shinkai R, Sakai S, Rughm JD, Paunovich ED (2001). Determinants of masticatory performance in dentate adults. Arc Oral Biol, 46(7): 641-648.

Javali SB, Parameshwar VP (2008). Use of the generalized linear models in data related to dental caries index. Indian J Dent Res, 18(4): 163-167.
Khader YS, Dauod AS, El-Qaderi SS, Alkafajei A, Batayha WQ (2006). Periodontal status of diabetics compared with non-diabetics: a meta-analysis. J Diabetes Complications, 20(1): 59-68.

Kwok T, Yu C, Hui H, Kwan M, Chan V (2004). Association between functional dental state and dietary intake of Chinese vegetarian old age home residents. Gerodontol, 21(3): 161-166.

Lalla E, Park DB, Papapanou PN, Lamster IB (2004). Oral disease burden in northern Manhattan patients with diabetes mellitus. Am J Pub Health, 94(5): 755-758.

Leong P, Tumanyan S, Blicher B, Yeung A, Joshipura K (2007). Periodontal disease among adult, new-immigrant, Chinese Americans in Boston with and without diabetes - a brief communication. J Pub Health Dent, 67(3): 171-173.

Lewsey J, Thomson W (2004). The utility of the zeroinflated Poisson and zero-inflated negative binomial models: a case study of cross-sectional and longitudinal DMF data examining the effect of socioeconomic status. Community Dent Oral Epidemio, 32(3): 183-189.

Mealey BL, Ocampo GL (2007). Diabetes mellitus and periodontal disease. Periodontol 2000, 44: 127-153.

Ministry of health, labour and welfare. Survey of diabetes mellitus. (2002). [WWW.document]. URL http://www. mhlw.go.jp/ shingi/2004/03/s0318-15.html-50.

Namariyama Y (2008). Relationship between community periodontal index and serum antibody levels for periodontopathicm microorganisms in a rural population in Kagoshima prefecture. J Dent Hlth, 58(1): 44-50.

Nelson RG (2008). Periodontal disease and diabetes. Oral Diseases, 14(3): 204-205.

Nishimura F, Iwamoto Y, Soga Y (2007). The periodontal host response with diabetes. Periodontology 2000, 43: 245-253.

Ogunbodede E, Fatusi O, Akintomide A, Kolawole K, Ajayi A (2005). Oral health status in a population of Nigerian diabetics. J Contemp Dent Pract, 6(4): 75-84.

Ohtake T, Takahashi R, Ohyabu Y, Minamisono N, Kuzuyama T, Ohishi K, Nagata T (2005). A study on community periodontal index of treatment needs (CPITN) in type 2 diabetic patients. J Jpn Soc Periodontol, 47(1): 28-35.

Shinkai R, Hatch J, Sakai S, Mobley CC, Saunders MJ, Rugh JD (2001). Oral function and diet quality in a community-based sample. J Dent Res, 80(7): 16251630. 
Smyth S, Heron A (2006). Diabetes and obesity: the twin epidemics. Nature Med, 12(1): 75-80.

Southerland JH, Taylor GW, Moss K, Beck JD, Offenbacher S (2006). Commonality in chronic inflammatory diseases: periodontitis, diabetes, and coronary artery disease. Periodontology 2000, 40: 130-143.

Statistical analysis committee on the survey of dental diseases (2005). Comprehensive guide to the survey of dental diseases. Tokyo: Oral Health Association.

Taylor GW, Borgnakke WS (2008). Periodontal disease: associations with diabetes, glycemic control and complications. Oral Diseases, 14(3): 191-203.
Tsai C, Hayes C, Taylor GW (2002). Glycemic control of type 2 diabetes and severe periodontal disease in the US adult population. Community Dent Oral Epidemiol, 30(3): 182-192.

Ueno M, Yanagisawa T, Shinada K, Ohara S, Kawaguchi Y (2010). Category of functional tooth units in relation to the number of teeth and masticatory ability in Japanese adults. Clin Oral Invst, 14(2): 113-119.

Ueno M, Yanagisawa T, Shinada K, Ohara S, Kawaguchi Y (2008). Masticatory ability and functional tooth units in Japanese adults. J Oral Rehabil, 35(5): 337-344.

\section{*Corresponding author: Masayuki Ueno}

Address: 1-5-45 Yushima, Bunkyo-ku, Tokyo 113-8549, Japan

Tel: $81358035477 \quad$ Fax: $81358030194 \quad$ E-mail: ueno.ohp@tmd.ac.jp 